Voix et Images

voixetimages

\title{
LA BANDE DESSINÉE QUÉBÉCOISE
}

Sous la plume des pionniers et des contemporains

CARMÉLIE JACOB et CATHERINE SAOUTER

Volume 43, numéro 2 (128), hiver 2018

La bande dessinée québécoise

URI : https://id.erudit.org/iderudit/1045061ar

DOI : https://doi.org/10.7202/1045061ar

Aller au sommaire du numéro

Éditeur(s)

Université du Québec à Montréal

ISSN

0318-9201 (imprimé)

1705-933X (numérique)

Découvrir la revue

Citer ce document

JACOB, C. \& SAOUTER, C. (2018). LA BANDE DESSINÉE QUÉBÉCOISE : sous la plume des pionniers et des contemporains. Voix et Images, 43(2), 7-13.

https://doi.org/10.7202/1045061ar d'utilisation que vous pouvez consulter en ligne.

https://apropos.erudit.org/fr/usagers/politique-dutilisation/ 


\title{
LA BANDE DESSINÉE QUÉBÉCOISE
}

Sous la plume des pionniers et des contemporains

\author{
$+++$ \\ CARMÉLIE JACOB \\ Université du Québec à Montréal \\ avec la collaboration de \\ CATHERINE SAOUTER \\ Université du Québec à Montréal
}

En 2008, Mira Falardeau écrivait dans son Histoire de la bande dessinée au Québec que, si l'un des objectifs du panorama de la bande dessinée locale qu'elle avait dressé quinze ans plus tôt ${ }^{1}$ était de "faire sortir de l'ombre cet art méconnu», il lui fallait «admettre que peu de choses [avaient] changé depuis et que les BD franco-belges, américaines et japonaises pren[aient] encore presque toute la place dans les librairies et les bibliothèques ${ }^{2} »$. Le Québec devait-il donc rester à jamais un peuple sans littérature bédéistique?

L'effervescence qu'a connue le neuvième art dans la province au cours de la dernière décennie prouve qu'il n'en est rien. Il n'est plus nécessaire de se rendre dans une librairie spécialisée pour avoir accès à la bande dessinée québécoise: les œuvres signées Jimmy Beaulieu, Chester Brown, Cathon, Julie Doucet, Jean-Paul Eid, Réal Godbout, Philippe Girard et Zviane - pour ne mentionner que quelques noms trouvent une place de choix dans la plupart de nos librairies, sans compter celles de Guy Delisle et de Michel Rabagliati, dont on voit de hautes piles jusque dans les grandes surfaces. Les institutions culturelles ne manquent d'ailleurs pas de souligner ce foisonnement, qu'on pense aux fréquentes chroniques que nous offrent Fabien Deglise (Le Devoir) et Jean-Dominic Leduc (Bande à part, Journal de Montréal, Les Libraires et la baladodiffusion Vous avez dit $B D$ ?) ou à l'émission de télévision $B D Q C$, diffusée sur ARTV. Des institutions qui, il n'y a pas si longtemps, semblaient refuser de voir en la bande dessinée une forme d'art digne de leur attention lui ouvrent maintenant fièrement leurs portes: avant même que le Musée québécois de culture populaire ne lui prête ses murs à Trois-Rivières ${ }^{3}$, le neuvième art québécois avait déjà en 2013 fait l'objet d'une exposition au Musée des beaux-arts de Montréal qui soulignait les quinze ans des éditions La Pastèque.

Quinze ans, voilà qui peut sembler court pour fêter un éditeur. Cette même année, Boréal célébrait ses cinquante ans. Les éditions Pierre Tisseyre existent depuis

1 Mira Falardeau, La bande dessinée au Québec, Montréal, Boréal, coll. «Boréal express», 1994, 125 p.

2 Mira Falardeau, Histoire de la bande dessinée au Québec, Montréal, VLB éditeur, coll. «Études québécoises», 2008, p. 9.

3 Exposition BDQ. L'art de la bande dessinée québécoise, 17 juin 2016 au 3 décembre 2018. 
1947; Fides, depuis 1937. Les quinze ans de La Pastèque, toutefois, marquaient bien ce qui semblait être l'accession à une pérennité nouvelle pour la bande dessinée francophone. Alors que la maison Drawn and Quarterly, née à Montréal en 1990, a connu - et connaît toujours - un grand succès grâce à la publication de bédéistes anglophones reconnus sur la scène internationale (tels que Lynda Barry, Chester Brown, Daniel Clowes, Kate Beaton, Seth, Art Spiegelman, Adrian Tomine et Chris Ware), les éditeurs de bande dessinée francophones ont longtemps eu du mal à se développer dans nos contrées.

Tout semblait pourtant possible lors des toutes premières publications du genre. Que l'on date cette naissance, comme le font Falardeau et la critique étatsunienne, à partir de l'utilisation du phylactère et d'un certain nombre de codes idéographiques (avec le Yellow Kid de Richard Outcault, paru en 1896) ${ }^{4}$, ou que l'on considère, comme Michel Viau et les Européens, que le récit en images fait partie intégrante de l'histoire de la bande dessinée (qui remonterait à 1827, avec l'Histoire de M. Vieux-Bois du Suisse Rodolphe Töpffer) ${ }^{5}$, on remarque que les expériences bédéistiques québécoises précèdent ces commencements. Viau attire par exemple l'attention sur un prospectus politique publié à l'été 1792 qui non seulement présente un récit en trois cases, mais fait déjà usage du phylactère ${ }^{6}$. Il s'agit là d'un cas isolé, certes, mais on retrouve ensuite dès le milieu du xix siècle, dans les périodiques humoristiques qui foisonnent, des gravures muettes ou légendées inspirées de l'œuvre de Töpffer sur le plan stylistique, mais qui s'intéressent aux affaires locales. Du reste, c'est au Québec qu'on voit apparaître les premières véritables bandes dessinées francophones avec phylactères. Après avoir publié quelques planches isolément aussi tôt qu'en 19027 , La Presse et La Patrie accueillent de 1904 à 1910, dans leur supplément du samedi, les œuvres des pionniers québécois que sont René-Charles Béliveau, Raoul Barré, Albéric Bourgeois et Théophile Busnel. Quoiqu'il soit fortement inspiré de celui des Américains et des Français, qui ne leur est pas étranger, leur travail rend compte de la société québécoise et permet aux lecteurs de ces journaux de s'y reconnaître. La série française de Christophe «La famille Fenouillard» (1889-1893) prend ainsi ce qu'il est opportun d'appeler une «saveur locale» lorsque René-Charles Béliveau l'adapte sous le titre de «La famille Citrouillard» et donne à ses personnages une langue qui n'a rien à envier à celle de Michel Tremblay: «Bonguenne! Quequ'c'est qu'ça que c't'animal-là8? ? s'écrie par exemple le père en voyant un train. Comme c'est souvent le cas avec les formes

4 Mira Falardeau, Histoire de la bande dessinée au Québec, p. 31.

5 Michel Viau, BDQ. Histoire de la bande dessinée au Québec, t. I: Des origines à 1979, Montréal, Mém9ire, 2014, p. 9-10.

6 Ibid., p. 16. Le prospectus en question, intitulé «Ā tous les électeurs», est reproduit dans le livre et mérite qu'on s'y attarde.

7 Viau mentionne même quelques cas exceptionnels de "prototypes de bandes dessinées à phylactères» publiés dès le milieu des années 1880 dans le journal satirique Le Canard, en précisant toutefois qu' «il s'agit de cas exceptionnels [puisque la] plupart des bandes dessinées de l'époque sont muettes ou légendées». Ibid., p. 25.

8 René-Charles Béliveau, «La famille Citrouillard», La Patrie, 30 avril 1904, archive disponible en ligne: http://www.collectionscanada.gc.ca/comics/027002-150-f.php?uid=027002-nlc002309\&uidc=recKey (page consultée le 20 janvier 2018). 
populaires, la bande dessinée de l'époque offre un portrait de la société bien différent de celui que proposent la poésie, le thêâtre ou le roman dans un paysage littéraire encore dominé par la langue des classiques français et les thèmes valorisés par le clergé. «Les contes du père Rhault» (Barré), les «Voyages du père Ladébauche» (Bourgeois) et «Le père Nicodème» (Béliveau) témoignent d'un catholicisme ambiant légèrement travesti, tandis que "Les fables du parc Lafontaine» et «L'histoire du Canada pour les enfants", deux séries de Bourgeois, ancrent résolument la forme dans le contexte québécois. Au total, entre 1904 et 1909, plus de six cents planches originales seront publiées dans La Presse et La Patrie, un rythme de production qui ne s'est jamais revu dans les quotidiens québécois depuis?.

Les œuvres locales disparaissent toutefois graduellement entre 1908 et 1910 avec l'expansion des syndicates américains, qui, en vendant les mêmes planches à un large éventail de journaux, parviennent à leur offrir des prix qui rendent vaine toute compétition. Sous la pression cléricale, on voit ensuite apparaître davantage de productions françaises, donc d'histoires en images, sans phylactères, et de bandes dessinées morales ou instructives pour la jeunesse qui imitent le style européen. En 1919, la Société Saint-Jean-Baptiste publie ses Contes historiques, d'abord dans sa revue L'oiseau bleu, puis dans des recueils dont les tirages atteindront 400000 exemplaires ${ }^{10}$. On voit bien émerger des créations pour la jeunesse qui ne sont pas commandées par le clergé, comme les récits avec phylactères d'Yvette Lapointe - l'auteure du premier strip quotidien québécois et la première femme à publier ses bandes dans un périodique ${ }^{11}$-, mais, même sous un titre comme "Petits espiègles", les personnages n'ont rien de l'insolence des Max et Moritz de Wilhelm Busch (Allemagne, 1865) ou des Katzenjammer Kids de Rudolph Dirks (États-Unis, 1897), dont ils semblent être inspirés.

La bande dessinée connaît ensuite «la période la plus sombre de son histoire ${ }^{12}$ » au cours des années 1930 à 1960, période pendant laquelle elle sera principalement employée à des fins idéologiques - lors de la Deuxième Guerre notamment, où elle louange la conscription et ridiculise le parti nazi. On assiste toutefois à cet événement marquant qu'est la création du populaire "Onésime» d'Albert Chartier, une chronique du quotidien et de la vie à la campagne publiée dans le Bulletin des agriculteurs, qui a eu la bonne idée, en 1943, de rejeter les syndicates pour faire appel à un bédéiste local. La série, d'une impressionnante qualité graphique et narrative, perdure jusque dans les années 1990, alors que le Québec a déjà connu, dans les années 1970, ce qu'on appelle son «Printemps de la BDK», qui désigne la naissance d'une institution.

$\mathrm{Si}$, à la mort de Duplessis, les lecteurs de BD québécois se tournent dans un premier temps vers les productions culturelles étrangères, on voit apparaître au cours de la décennie 1970 une multitude de fanzines et de revues. Ceux-ci, sous l'influence de l'underground étatsunien et des périodiques franco-belges, se consacrent entièrement

9 Michel Viau, BDQ, t. I: Des origines à 1979, p. 50.

10 Ibid., p. 64.

11 Ibid., p. 101.

12 Mira Falardeau, Histoire de la bande dessinée au Québec, p. 65. 
à la bande dessinée $\left(M a{ }^{\circledR}\right.$ de in Kébec, BD, L’hydrocéphale illustré, Kébec poudigne, L'illustré, L'écran, Plouf, Baloune) ou lui font une place de choix parmi des articles ou des nouvelles littéraires (Mainmise, Requiem - devenue Solaris -, Québec-Presse, Prisme). Ces publications ne produisent le plus souvent que quelques numéros, mais elles permettent aux bédéistes de se rencontrer, d'échanger et de se regrouper. Au fil des expositions et des congrès, le milieu devient suffisamment dynamique pour mener à la naissance du magazine Croc (1979-1995), qui fera connaître des bédéistes d'importance comme Lucie Faniel, Serge Gaboury, Michel Garneau (Garnotte), Réal Godbout et Jean-Paul Eid. Plusieurs de ces auteurs travailleront, parallèlement ou postérieurement, pour d'autres périodiques (Titanic ${ }^{13}$, Safarir et Délire, notamment), mais Croc donne aussi à la BD une poussée suffisamment forte pour que la constitution d'albums soit enfin possible.

Sous cette impulsion, on voit apparaître en 1989 les Éditions Mille-Îles, qui deviennent cinq ans plus tard Les 400 coups (dont la filiale Mécanique générale, autrefois maison d'édition de Jimmy Beaulieu, est entièrement consacrée à la forme bédéistique) et donnent le coup d'envoi à d'autres éditeurs spécialisés, tels que L'Oie de Cravan (1992), La Pastèque (1998) ou Colosse (2002). Comme les coûts d'impression de la $\mathrm{BD}$ ont toujours été un des principaux freins à sa diffusion, l'arrivée d'Internet a grandement participé à la revitalisation du milieu: les bédéistes, qui dans les années 1970 se rencontraient lors d'événements, communiquent maintenant au sein de la blogosphère, qui permet à l'artiste de montrer son travail à moindres frais, mais surtout d'obtenir rapidement des commentaires d'autres artistes et de tisser des liens. Avant même d'être publié, l'auteur a souvent un important bassin de lecteurs - pensons à Iris Boudreau ou à Zviane, qui se sont fait connaître grâce à leur blogue dès le début du deuxième millénaire - , et est par conséquent plus attrayant pour les éditeurs. Ce nouveau mode de diffusion, qui profite maintenant de la visibilité offerte par les réseaux sociaux comme Facebook et Instagram, n'est sans doute pas étranger à l'éclosion, ces dernières années, de nombreuses maisons d'édition de qualité consacrées exclusivement à la bande dessinée: BerBer, La mauvaise tête, Pow Pow, Studio Lounak, Trip, etc.

Michel Viau observe avec dépit que la période marquée par l'arrivée des planches de Bourgeois, de Barré, de Béliveau et de Busnel dans La Presse et La Patrie est «bien souvent qualifiée d'âge d'or de la bande dessinée québécoise» - «comme si la période la plus riche de son histoire était également celle de sa naissance ${ }^{14}$ ", ajoute-t-il avec raison. Nous croyons au contraire que l'édition de la bande dessinée au Québec n'a jamais été aussi féconde qu'aujourd'hui. En témoigne le succès public de la série de Rabagliati et du film inspiré de Paul à Québec, mais aussi l'omniprésence, dans les librairies, à la télé, dans les journaux, à la radio, de cette forme qui semblait encore, dans les années 1990, réservée aux plaisirs de l'enfance ou à l'humour facile.

13 Sur l'apport important de cette revue au champ de la bande dessinée québécoise, voir Catherine SaouterCaya, La bande dessinée québécoise (1979-1984). Éléments pour une sémiologie de la bande dessinée, thèse de doctorat, Montréal, Université du Québec à Montréal, 1990, 337 f.

Michel Viau, BDQ, t. I: Des origines à 1979, p. 50. 
Les institutions culturelles reconnaissent de plus en plus le caractère hétéroclite de cette forme, qui - il faut y insister - n'est pas en soi un genre, mais au contraire appelle tous les genres et se modèle à eux. Néanmoins, il nous a semblé qu'il restait encore un domaine où la bande dessinée québécoise ne recevait pas toute l'attention qu'elle méritait, soit celui de la critique savante. Il y a bien eu, dans les années soixante-dix à quatre-vingt-dix, un intérêt marqué pour la BD dans le monde universitaire. André Carpentier, Jacques Samson, Richard Langlois, par exemple, ont été parmi les premiers à faire connaître la bande dessinée au cégep et à l'université, et La Barre du jour lui consacrait déjà au début des années 1970 un numéro quadruple, qui analysait le phénomène au moment même où il se produisait ${ }^{15}$. Près de cinquante ans plus tard, pourtant, les choses semblent avoir bien peu bougé: les travaux savants sur la bande dessinée québécoise se font encore rares.

Ainsi, on ne s'étonnera pas trop que ce dossier soit le premier que Voix et Images consacre à la bande dessinée québécoise. Dans ce contexte que l'on peut dire inaugural, nous proposons un portrait de quelques productions contemporaines, qui met de l'avant certains phénomènes, comme l'emprunt aux genres littéraires (l'autobiographie, l'essai) et la référence aux beaux-arts. Ce premier échantillon des éléments narratifs et graphiques de la bande dessinée contemporaine est par ailleurs précédé d'un regard sur la naissance du média dans la presse locale, ce qui permet de constater le travail accompli pendant le siècle dernier. Nous espérons ainsi ouvrir un champ de recherches qui donnera lieu à l'exploration de cette histoire finalement peu connue de la bande dessinée d'ici.

Ce projet de faire entrer le neuvième art dans la revue Voix et Images n'aurait pas été tout à fait achevé sans l'œuvre qu'a spécialement conçue Jean-Paul Eid pour la couverture de ce numéro. Bédéiste éclectique, Eid a marqué la bande dessinée québécoise dès le milieu des années 1980 par ses contributions au magazine Croc, et il continue de la réinventer avec des œuvres aussi distinctes que Le fond du trou (2011) et La femme aux cartes postales (2016; coscénarisée avec Claude Paiement). L'œuvre qu'il a créée pour ce dossier non seulement témoigne de son habituelle aisance à jouer avec les codes, mais donne à voir, si l'on peut dire, les questions qui sont abordées dans ces pages, notamment l'importance accordée à la relation de l'auteur à son travail — l'intérêt actuel pour l'autobiographie étant pratiquement omniprésent — et le métalangage associé à la valeur rythmique de la case.

Au Québec, le langage bédéistique s'est développé dans des planches publiées dans La Presse et La Patrie au début du $\mathrm{xx}^{\mathrm{e}}$ siècle, et il nous semblait essentiel que le dossier s'ouvre en traitant de cette genèse méconnue. C'est donc ce moment que dépeint Stéphanie Danaux, en suivant les traces du dessinateur breton Théophile Busnel, qui s'est installé à Montréal entre 1904 et 1908 et a rapidement su se mêler aux autres pionniers que sont Raoul Barré, René-Charles Béliveau et, surtout, son collègue et ami Albéric Bourgeois, dont il reprendra le célèbre personnage de Timothée. L'article témoigne d’un début éclatant pour la bande dessinée québécoise,

15 Voir André Carpentier (dir.), La bande dessinée kébécoise (Bois-des-Filion, La Barre du Jour, 1975, 260 p.), qui regroupe les numéros 46 à 49 de la revue. 
qui dépeignait déjà les mœurs locales d'une façon que les autres formes littéraires mettraient encore des décennies à découvrir.

Un siècle plus tard, où en est la bande dessinée? C'est la question à laquelle répond Mario Beaulac, dans un article où il esquisse un portrait de cet art aujourd'hui, à partir du métadiscours sur la bande dessinée et des œuvres majeures de Rabagliati, d'Obom, de Zviane et de Beaulieu. En travaillant sur ces deux derniers auteurs, majeurs non seulement en raison de leur œuvre, mais aussi de leur apport au milieu en tant qu'éditeurs (La jungle, Mécanique générale...) et professeurs, il pose d'ailleurs les balises des deux articles qui closent le dossier. On y constate, évidemment, que la BD est bien loin des courtes histoires humoristiques de nos pionniers, la réflexion sur le média ayant depuis été alimentée non seulement par des œuvres comme «Onésime» et le milieu BDKesque foisonnant des décennies 1970 à 1990, mais aussi par le graphic novel étatsunien et les expérimentations de Français qui gravitent autour d'éditeurs indépendants tels que L'Association.

Un tour d'horizon d'un tout autre ordre est ensuite proposé par Catherine Saouter, qui s'intéresse pour sa part à un événement: «La BD s'expose au musée», exposition présentée au Musée des beaux-arts de Montréal du 6 novembre 2013 au 30 mars 2014, qui proposait aux bédéistes de La Pastèque de créer quelques planches inspirées d'œuvres d'art de sa collection. L'article décrit les différentes techniques dont ont usé les bédéistes pour s'approprier une œuvre et l'intégrer à leur bande dessinée sans renier ce qui fait leur style. Parallèlement, il révèle que les liens entre musée et bande dessinée sont plus fréquents qu'on le croirait d'emblée, la frontière entre ce qui était autrefois classifié en «culture haute» et en «culture basse» tendant enfin à s'effacer.

L'article qui suit, celui de Jean-Philippe Beaulieu, se penche justement sur une œuvre où Vélasquez et Picasso sont à l'honneur, soit Tuer Vélasquez de Philippe Girard, qui aborde de façon autobiographique la question sensible de la pédophilie au sein du clergé. Il s'intéresse plus spécifiquement à la façon dont le caractère autoréférentiel de l'œuvre se manifeste, la bande dessinée se distinguant de la plupart des productions associées à ce genre en ce que

\begin{abstract}
le discours autobiographique semble moins autotélique: bien qu'il occupe une place centrale dans le déploiement du récit, il attire relativement peu l'attention sur luimême et présente la subjectivité de Philippe de manière plutôt indirecte, par le truchement d'un réseau de références qui viennent enrichir une matière narrative dont la trame est relativement simple ${ }^{16}$.
\end{abstract}

Beaulieu parvient ainsi à montrer comment un sujet aussi sensible peut être abordé à partir de l'expérience personnelle - d'une expérience personnelle - sans passer par le pathos.

Gabriel Tremblay-Gaudette s'intéresse quant à lui à l'œuvre largement autobiographique de Jimmy Beaulieu; mais il s'agit d'une forme d'autobiographie qui

16 Voir, dans le présent dossier, Jean-Philippe Beaulieu, «"N’hésitez pas à jouer la carte de l'histoire vécue." Les demi-teintes de l'autobiographie dans Tuer Vélasquez de Philippe Girard», p. 59. 
s'oppose justement à celle de Girard, et qui sert à raconter, de façon très introspective, les événements les plus anodins de la vie quotidienne. Sans prendre appui sur une monographie particulière, les livres de Beaulieu se lisant pour la plupart comme des fragments d'une seule et même longue œuvre, Tremblay-Gaudette met au jour que la méthode autobiographique de l'auteur repose sur une fidélité absolue au pacte de vérité qu'il scelle avec son lecteur, laquelle justifie qu'il se mette à nu et n'hésite pas à laisser entrevoir ses failles.

Enfin, Carmélie Jacob rend compte d'un genre qui, comme le souligne son article, n'est pas sans parenté avec les écritures autobiographiques, mais se détache du sujet pour se concentrer sur un objet culturel: l'essai, genre encore marginal en bande dessinée, qu'elle aborde à travers la version commentée du Ping-pong de Zviane. En faisant un détour par les théories de l'essai, elle signale de quelle manière l'œuvre emploie les codes du genre, avant de s'intéresser à la nature polyphonique de la version commentée publiée chez Pow Pow, qui consiste en une surimposition, à l'œuvre originale publiée à compte d'auteur, des commentaires de Zviane et de dixneuf bédéistes invités. L'article révèle qu'à travers Ping-pong, l'auteure explore une nouvelle façon de conférer à l'essai le caractère ouvert qu'il cherche inévitablement à atteindre.

On remarquera que ce dossier se présente en quelque sorte comme un chemin de fer dont on n'aurait pour le moment que construit les gares, et une section de rails à l'arrivée. Il est indéniablement troué. Ce manque est attribuable d'une part à ce qui a été révélé dans le bref historique que nous avons fait en début de parcours, soit au fait que la tradition bédéistique québécoise se présente elle-même comme un chemin de fer dont on n'aurait construit que des pans. Entre les années 1910 et 1970, la production n'est pas inexistante, mais elle est restreinte. D'autre part, et pour la section chronologique qui sépare 1970 de 2000, le manque est en quelque sorte inverse: cette période a connu un nouveau bouillonnement en bande dessinée, à la fois sur le plan artistique et sur le plan critique. Un petit groupe de chercheurs fort prolifique s'est intéressé à cette période, sur laquelle il est plus aisé d'accéder à des ressources théoriques que sur les débuts du genre et la production hypercontemporaine. Les propositions que nous ont soumises nos collaborateurs ont rapidement révélé une soif de travailler sur ce qui constitue des terrains pratiquement indéfrichés dans la critique savante sur la bande dessinée, et il nous a semblé que cette soif serait aussi celle des lecteurs de ce numéro. La bibliographie préparée par Jean-Michel Berthiaume, qui figure en fin de dossier, permettra en outre aux lecteurs de repérer facilement les principaux articles et ouvrages monographiques qui s'intéressent à l'histoire de la bande dessinée d'ici. Cette bibliographie critique et théorique montre que le sujet est loin d'être saturé, et les chercheurs ne manquent pas de matériau à explorer. Il ne tient qu'à nous de prolonger cette voie. 